Open Peer Review on Qeios

\title{
Enabling countries to apply behavioural science in using global survey data to inform their Covid-19 policies
}

\author{
Elizabeth Corker $^{1}$, Elena Altieri² ${ }^{2}$ Susan Michie ${ }^{1}$ \\ 1 University College London, University of London \\ 2 World Health Organization WHO
}

Funding: The author(s) received no specific funding for this work.

Potential competing interests: The author(s) declared that no potential competing interests exist.

\begin{abstract}
The COVID-19 pandemic has highlighted the vital importance and impact of human behaviour on viral transmission. During 2020, large amounts of global survey data were collected and made freely available to help the response. Many teams responding to the pandemic lack capacity to identify and interpret behavioural data. A collaboration of the World Health Organization's Behavioural Insights team and UCL's Centre for Behaviour Change designed and piloted two templates to enable survey data use during the first months of the pandemic. The first template documents key behaviours, thoughts and emotions related to the pandemic, along with social interactions and population adherence to behavioural guidelines. The second template enables countries to formulate questions or issues that they would like behavioural data to address. This collaborative process applying behavioural science theory produced structured templates to enable organisation and interpretation of survey data to inform policy and practice in different country contexts.
\end{abstract}

\section{Introduction}

The December 2019 outbreak of the respiratory virus SARS-CoV-2, which causes COVID-19 (Li et al., 2020) led to its characterisation as a global pandemic by the World Health Organization (WHO) in March 2020 (World Health Organization, 2020a). Human behaviour is at the heart of viral transmission (Michie \& West, 2020a). It is also at the heart of breaking that transmission through effective delivery of public health measures, clinical management of patients, and deployment of vaccines and treatment (Flaxman et al., 2020; Islam et al., 2020; World Health Organization, 2020b).

The development and nature of the COVID-19 crisis can be described as a 'wicked problem'. A 'wicked problem' is difficult to resolve due to incomplete or contradictory understandings, differing and changing contextual requirements and difficulties in recognising the problem's components (Rittel \& Webber, 1973). 
There is usually no single solution to wicked problems, and they require expertise from multiple disciplines to find a way forward (Rittel \& Webber, 1973). As such, long-term resolutions to wicked problems involve multiple complex processes and may be undermined by a lack of understanding regarding vital aspects of the problem, such as human behaviour (Michie et al., 2015).

Understanding the transmission of COVID-19 and how best to suppress it is a global priority. Developing this understanding requires collecting, analysing and acting on large amounts of data referring to different aspects of the population, their everyday behaviours and their social and economic contexts (Michie \& West, 2020). A vast number of surveys are being conducted, recording COVID-19 related perceptions, knowledge, beliefs and behaviours of populations globally (e.g. (Bacon et al., 2021; Kantar, 2020; YouGov, 2020)). Attempts are also ongoing to track survey data relating to COVID-19 (Matias \& Leavitt, 2020). Considerable financial and human resources are invested into the collection and production of survey data aimed at understanding behaviours in the context of the pandemic. Much data produced has been made freely available to support policy teams and governmental and other organisations in their responses to COVID-19.

Behavioural data are potentially of great use to policy-makers, planners, practitioners and researchers focussed on pandemic response. Data collection is only one aspect of the complex task of a behaviourally informed public health response. Partially due to the scale and speed of data becoming available, there has been little to guide and support in-country stakeholders, including local WHO teams responding to the pandemic, to interpret and operationalise survey data collected and made available by a variety of entities such as universities or polling agencies. Increasing the data interpretation and operationalisation capacity of stakeholders can increase local understanding of behavioural patterns and the effectiveness of responses. Without the means to interpret the large amounts of behavioural data being generated, there is a risk of it not being used to inform and improve responses or being misinterpreted (Flaxman et al., 2020; Islam et al., 2020; World Health Organization, 2020b).

Tools, such as frameworks and templates for structuring information, provide a way of organising data and can be used to identify links between research findings, theory and responses. Tools can make data more accessible and useable for policy-makers and practitioners in a range of country contexts. Templates have been used successfully by organisations such as the UK's National Institute for Health and Care Excellence (NICE) (National Institute for Health and Care Excellence, 2014). Templates also enable shared language to describe and apply behavioural data across datasets and stakeholders, improving communication between researchers and between researchers and knowledge users. The use of shared language can avoid confusion when the same term describes different things (concepts or constructs) or when several terms describe the same thing.

Theories of behaviour and behaviour change summarise what we know about how behaviour is influenced by, and influences, thoughts, emotions and people's social and material environments. Using theory-based tools to interpret behavioural data can provide descriptions and explanations of influences on and outcomes of behaviours. They can also help to generate testable hypotheses about associations between 
behaviours and other factors (Davis et al., 2015; Michie et al., 2014).

A widely used model of behaviour is COM-B (Capability, Opportunity, Motivation and Behaviour) (Michie et al., 2011; West \& Michie, 2020). The COM-B model suggests that behaviour is a result of a person's capability (both psychological and physical) to perform the behaviour, the opportunity (both physical and social) to carry out the behaviour and the motivation (both automatic and reflective) to carry out the behaviour (see Figure 1). The model highlights that behaviour is a complex process and the result of an interaction of factors, rather than a linear progression from knowledge through to intentions and behaviour (Sniehotta et al., 2005).

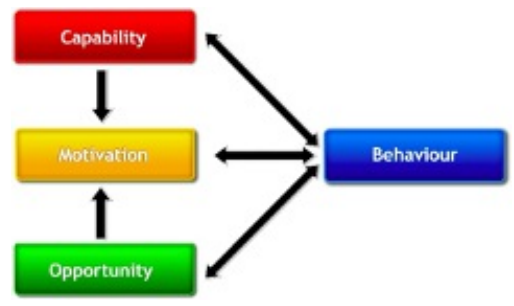

Figure 1. The COM-B model of behaviour. Taken from Michie, S. et al 2011.

The COM-B model forms the hub of the Behaviour Change Wheel, a framework of interventions and policies, which links to a taxonomy of 93 specific behaviour change techniques (Michie et al., 2013; Michie et al., 2011). These tools have been used to inform the development and evaluation of interventions and policies and to synthesise evidence about effectiveness (Chadwick et al., 2020; Gould et al., 2019; Public Health England, 2018; The British Psychological Society, 2019; Webb et al., 2016). Associated with the Behaviour Change Wheel is a set of criteria to guide implementation, termed APEASE which stands for Acceptability, Practicability, Effectiveness, Affordability, Spillover and Equity (Michie et al., 2013). An example of applying these criteria was in the UK Government's Scientific Advisory Group in Emergencies report evaluating options for COVID-19 related restrictions, such as physical distancing (Michie et al., 2020). This work was aided by the use of a structured template.

It is imperative to consider the range of actions available to enable the behaviour changes and actions required when forming a pandemic response. An essential part of a pandemic response is communication (Hyland-Wood et al., 2021). However, providing information is not sufficient in isolation. Behavioural data can be used to inform a range of actionable recommendations. Theory-based templates designed using a collaborative approach, combining behaviour science and communications expertise could help countries focus on issues of most concern and to access survey data in a way that relates to their specific context to inform actionable recommendations.

\section{Aim and Objectives}

This work aimed to combine behavioural science and communication expertise to develop templates usable within a global context that enable the extraction, synthesis and interpretation of behavioural 
survey data in a way that are maximally useful and useable to a range of countries and audiences.

The objectives were to:

1. Develop a theory-based, data organisation template.

2. Develop a template to assist country teams to identify additional survey questions to provide data to inform their policy and practice.

\section{Methods}

This work was produced by a collaboration between the Centre for Behaviour Change and the Behavioural Insights Initiative Team in the Department of Communications at WHO. Between April and June 2020, we designed two theory-based templates: one to organise data, the Survey Findings Template, and the other to identify additional survey questions, the Additional Survey Items Template. The templates were populated using data from international surveys. We piloted the templates with intended end-users (endusers were WHO staff in regional and country offices including field workers, emergency and technical advisors, and communication team members, along with local partners). We provided training to use the templates through webinars to end-users.

\section{Survey Findings Template}

We used COM-B components as an organising structure to develop a template with two sections to extract key data from international surveys. The first section recorded influences on behaviours reported in the data:

- Feelings (for example, emotional responses to risk).

- Thoughts (for example, beliefs or perceptions of risk).

- Behaviours (for example, health-related behaviour or media consumption).

The second section recorded data relating specifically to influences (capability, motivations and opportunity) impacting adherence to government guidelines, as this was central to reducing transmission of COVID-19.

Data from international surveys were used to populate both sections of the Survey Findings Template for 11 countries: Algeria, Brazil, Chile, China, India, Malaysia, Nigeria, Peru, Philippines, Thailand and Vietnam. These countries were selected because they represented a geographical or contextual range, allowing for diverse learning in applications of the template, and there was interest within each country or regional team to use and act upon the data available.

\section{Additional Survey Items Template}

The Additional Survey Items Template acknowledged the limitations of data to answer specific questions 
that may be important to answer within local contexts. This template asked end-users to specify the questions they would like the data to answer.

\section{Piloting the Templates}

The Templates underwent a round of pilot testing with end-users from regional offices responsible for several countries. We sent completed templates to them for review, and they provided written comments and feedback in teleconference calls. We asked for feedback about how useable and useful they were and suggestions for additions or other changes. We used this feedback to refine the templates.

Several small revisions were made, along with one substantive addition suggested for the Survey Findings Template, relating to reported instances of stigma and discrimination in social situations within a population. Stigma is the attitudes and beliefs directed towards a person or group of people and discrimination relates to negative behaviour directed towards a person or a group of people (Thornicroft, 2006). To capture data recording these social interactions, we included an additional section:

- Social interactions: Data relating to instances of stigma or discrimination aimed at particular populations or groups.

\section{Results}

This collaborative piece of work resulted in two behaviour science theory-based templates. The Survey Findings Template and associated guidelines for use are available as Supplementary File 1. The Additional Survey Items Template and associated guidance are available as Supplementary File 2.

Figure 2 displays an illustrative example of each section of a populated Survey Findings Template. We do not have the rights to share the data from the international surveys, the figures throughout this article present fictional data. 


\section{How is the}

population:

Survey finding

Feeling (add rows as appropriate)

$45 \%$ of the population are worried about

Feeling 1 falling sick no matter what precautions they take.

\section{Context/ interpretation}

This country has high rates of COVID-19 morbidity and mortality compared to other countries in the region.

This country has an older than average population and the biggest source of employment in this country is factory work. There is some distrust regarding how the government has handled the pandemic.

\section{Application: Action point/s}

Ensure that national media communications acknowledge the levels of concern and provide clear messaging from trusted sources about the likelihood of being infected, including information for more vulnerable groups and steps the population can take to decrease that likelihood. Communications regarding measures related to health should be made via increasingly popular social media channels, such as WhatsApp and print media to ensure the information is not restricted to those with internet access.

Social Interactions (add rows as appropriate)

$36 \%$ of the

Social population report

interaction 1 concern regarding people returning

from trips to other countries.
There have been several reports in the media regarding 'super spreaders', along with contradicting reports regarding how the virus is transmitted.

Evidence suggests that adherence to travel guidelines is high.
Ensure that national media communication acknowledge levels of concern and provide clear messaging from trusted sources about the likelihood of being infected. Ensure that polices and guidance related to travel are clear and that measures are taken to ensure these measures can be followed.

Communications regarding measures related to travel should be made via increasingly popular channels such as WhatsApp and print media to ensure that the information is not restricted to those with internet access.

Communications should emphasize the high levels of adherence.

Adherence to government guidance (add row as appropriate).

Influences on adherence are categorised according to the COM-B model, see https://www.ucl.ac.uk/behaviour-change/abc-guides and https://www.youtube.com/watch?v=-6RvEF9L4|Y\&feature=youtu.be

\section{Adherence 1 \\ Increased \\ handwashing \\ Survey Finding: \\ Over time, the majority of those surveyed have reported increasing frequency of hand washing increases (from $79 \%$ to $89 \%$ ).}

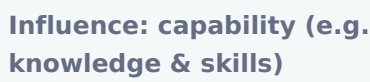

Influence: capability (e.g. knowledge $\&$ skills)

Use trusted media sources to maintain awareness of

Action point/s
Awareness of links between personal protective behaviours and virus spread. importance of personal
Influence: motivations

(e.g. emotions \& attitudes)

Concerns around catching COVID-19 remains high.

\section{Influence: opportunity (social \& physical)} protective behaviours and to demonstrate good practice.

Appeal to social norms regarding majority adhering to handwashing guidance.
Requires access to water and soap or hand sanitiser.

Figure 2. Illustrative example of the Survey Findings Template.

Figure 3 displays an illustrative example of a completed row of the Additional Survey Items Template, using fictional data. 
Your issue or

question (add rows Context of your issue or question How you would like to use the advice

as appropriate)

Issue/question 1:

What proportion of the population believes that good hand hygiene is important during a pandemic, and is there a difference between younger and older generations?

The country has an older population and the main source of employment is within factories.
We will use the advice to inform recommendations relating to the types and mechanisms of communication with the general public or within specific groups within the population.

Figure 3. Illustrative example of the Additional Survey Items Template.

\section{Discussion}

A collaborative process applied behavioural science theory to produce structured templates that facilitate the organisation and interpretation of survey data to inform practice and policy in different country contexts. The Survey Findings Template provides space to document key self-reported behaviours, thoughts and emotions concerning the pandemic. It also records social interactions and population adherence to behavioural guidelines. It also offers space for interpreting data within the local country context. The Additional Items Template enables countries to formulate questions or issues that they would like behavioural data to address.

Understanding global issues requires expertise from multiple disciplines (Rittel \& Webber, 1973). We presented and discussed the templates with teams who do not have behavioural science expertise. This work demonstrates how collaborations between different areas of expertise, in this case, behavioural science theory and effective pandemic response, can be beneficial for producing results that are usable within fast-changing global contexts such as pandemics. We recommend interdisciplinary ways of working in moving forward with complex global issues, which will improve formulations of issues and reduce the risk of overlooking aspects important to solutions (Rittel \& Webber, 1973).

The practical implications for this work include improving researchers and practitioners' understanding of how behavioural theory can support understanding of data aimed at capturing current and evolving issues. The Survey Findings Template allows end-users who may not be familiar with behavioural science to understand what influences behaviours, in terms of barriers and drivers, through the use of self-reported behavioural data. Additionally, the template enables users to frame influences on behaviour within their local context, thereby understanding what actions may benefit the local population. As new data emerge, this template can aid in understanding its relevance within the context of what is already known. Increasing capacity in data interpretation contributes to building a comprehensive picture of behaviour related to a particular issue. The Additional Survey Items Template allows end-users to develop their understanding of behavioural science and needs for specific behavioural data by identifying gaps in the data available and formulating questions for future research. 
A limitation of this work is that we did not have ownership of the survey data collected. Consequently, we were unable to influence the design of the surveys. Lack of data ownership may appear to reduce the usability of data. However, as we aimed to design templates that enable the extraction and interpretation of existing data, we were able to demonstrate how to maximise the use of available data in three ways: first, by identifying the key points, second by interpreting them using local knowledge and finally, operationalising them through actionable recommendations. Furthermore, we tested the templates on data from multiple countries, indicating generalisability and usability across contexts. Additionally, endusers of the templates are from different disciplines demonstrating usability from those without a behavioural science background. Overall, the templates enable theoretically informed recommendations to be accessible to a wide-ranging cohort of users.

Although we developed these templates in response to data concerning the COVID-19 pandemic, we regard them as relevant to other global issues such as climate change or health inequality. Future work could include collaborations with teams holding expertise in different disciplines such as climate science or health service design. Assessing the utility of data organisation templates based on behavioural science within different contexts will enable a deeper understanding of the impact of human behaviour.

The templates designed here will support better coherence and collaboration amongst researchers, practitioners and policy-makers. They will enable the development of realistic recommendations that are evidence-informed and translatable into real-world practice through the structured assessment of data highlighting: what the behavioural issue is, what is driving the behaviour and what would work well to address this within local contexts. The development of data organisation templates may contribute towards the global effort of enabling behavioural science to inform the management of 'wicked problems' (Rittel \& Webber, 1973).

\section{Funding}

SM received a small consultancy grant in March 2020 for this work from the World Health Organization. This grant was received prior to SM becoming a nominated member of the WHO Technical Advisory Group on Behavioural Insights and Sciences for Better Health in July 2020.

\section{Declarations of interest}

This grant for this work was received prior to SM becoming a nominated member of the WHO Technical Advisory Group on Behavioural Insights and Sciences for Better Health in July 2020.

\section{References}

Bacon, S. L., Lavoie, K. L., Boyle, J., Stojanovic, J., \& Joyal-Desmarais, K. (2021). International assessment of 
the link between COVID-19 related attitudes, concerns and behaviours in relation to public health policies: Optimising policy strategies to improve health, economic and quality of life outcomes (the iCARE Study). BMJ Open, 11(3). https://doi.org/10.1136/bmjopen-2020-046127

Chadwick, P., Pender, T., \& Onduru, E. (2020). Using the behaviour change wheel framework within genderfocused international development programmes: a Field Guide. https://actionaid.ie/wpcontent/uploads/2020/10/ActionAid-Behaviour-Change-Manual.pdf

Davis, R., Campbell, R., Hildon, Z., Hobbs, L., \& Michie, S. (2015). Theories of behaviour and behaviour change across the social and behavioural sciences: a scoping review. Health Psychology Review, 9(3), 323-344. https://doi.org/10.1080/17437199.2014.941722

Flaxman, S., Mishra, S., Gandy, A., Unwin, H. J. T., Mellan, T. A., Coupland, H., Whittaker, C., Zhu, H., Berah, T., Eaton, J. W., Donnelly, C. A., Riley, S. M., Vollmer, M. A. C., Ferguson, N. M., \& Okell, L. C. (2020). Estimating the effects of non-pharmaceutical interventions on COVID-19 in Europe. Nature, June, 1-8. https://doi.org/10.1038/s41586-020-2405-7

Gould, G. S., Bovill, M., Pollock, L., Bonevski, B., Gruppetta, M., Atkins, L., Carson-Chahhoud, K., Boydell, K. M., Gribbin, G. R., Oldmeadow, C., Hall, A., \& Bar-Zeev, Y. (2019). Feasibility and acceptability of Indigenous Counselling and Nicotine (ICAN) QUIT in Pregnancy multicomponent implementation intervention and study design for Australian Indigenous pregnant women: A pilot cluster randomised stepwedge trial. Addictive Behaviors, 90(August 2018), 176-190. https://doi.org/10.1016/j.addbeh.2018.10.036

Hyland-Wood, B., Gardner, J., Leask, J., \& Ecker, U. K. H. (2021). Toward effective government communication strategies in the era of COVID-19. Humanities and Social Sciences Communications, 8(1), 1-11. https://doi.org/10.1057/s41599-020-00701-w

Islam, N., Sharp, S. J., Chowell, G., Shabnam, S., Kawachi, I., Lacey, B., Massaro, J. M., Sr, R. B. D. A., \& White, M. (2020). Physical distancing interventions and incidence of coronavirus disease 2019 : natural experiment in 149 countries. British Medical Journal. https://doi.org/10.1136/bmj.m2743

Kantar. (2020). Kantar's COVID-19 National Barometer.

https://www.kantar.com/Inspiration/Coronavirus/COVID-19-Barometer-Consumer-attitudes-media-habitsand-expectations

Li, Q., Guan, X., Wu, P., Wang, X., Zhou, L., Tong, Y., Reb, R., Leung, K. S. M., Lau, E., Wong, J. Y., Xing, X., Xiang, N., Wu, Y., Chao, L., Chen, Q., Li, D., Liu, T., Zhao, J., Liu, M., ... Feng, Z. (2020). Early transmission 
dynamics in Wuhan, China, of novel Coronavirus-infected pneumonia. The New England Journal of Medicine, 382(13), 1199-1207. https://doi.org/10.1056/NEJMoa2001316

Matias, N., \& Leavitt, A. (2020). COVID-19 International Academic Social Science Research Project Tracker. https://github.com/natematias/covid-19-social-science-research/

Michie, S., Richardson, M., Johnston, M., Abraham, C., Francis, J., Hardeman, W., Eccles, M., Cane, J., \& Wood, C. E. (2013). The behavior change technique taxonomy (v1) of 93 hierarchically clustered techniques: Building an international consensus for the reporting of behavior change interventions. Ann Behav Med, 46(1), 81-95. https://doi.org/10.1007/s12160-013-9486-6

Michie, S., Stralen, M. M. Van, \& West, R. (2011). The behaviour change wheel: A new method for characterising and designing behaviour change interventions. Implementation Science, 6(42).

https://doi.org/https://doi.org/10.1186/1748-5908-6-42

Michie, S., \& West, R. (2020). Behavioural, environmental, social, and systems interventions against covid19. BMJ, 370. https://doi.org/10.1136/bmj.m2982

Michie, S., West, R., Capbell, R., Brown, J., \& Gainforth, H. (2014). ABC of Behaviour Change Theories: An Essential Resource for Researchers, Policy Makers and Practitioners. Silverback Publishing.

Michie, S., West, R., Rogers, M. B., Bonell, C., Rubin, G. J., \& Amlôt, R. (2020). Reducing SARS-CoV-2 transmission in the UK: A behavioural science approach to identifying options for increasing adherence to social distancing and shielding vulnerable people. British Journal of Health Psychology, 25(4), 945-956. https://doi.org/10.1111/bjhp.12428

Michie, S., Wood, C. E., Johnston, M., Abraham, C., Francis, J. J., \& Hardeman, W. (2015). Behaviour change techniques: The development and evaluation of a taxonomic method for reporting and describing behaviour change interventions (a suite of five studies involving consensus methods, randomised controlled trials and analysis of qualitative da. Health Technology Assessment, 19(99), 1-187.

https://doi.org/10.3310/hta19990

National Institute for Health and Care Excellence. (2014). Developing NICE guidelines: the manual. http://www.nice.org.uk/article/pmg20

Public Health England. (2018). Improving people's health: Applying behavioural and social sciences to improve population health and wellbeing in England. 
https://assets.publishing.service.gov.uk/government/uploads/system/uploads/attachment_data/file/744672/l mproving_Peoples_Health_Behavioural_Strategy.pdf

Rittel, H., \& Webber, M. (1973). Dilemmas in a general theory of planning. Policy Sciences, 4(2), 155-169. https://doi.org/https://doi.org/10.1007/BF01405730

Sniehotta, F. F., Scholz, U., \& Schwarzer, R. (2005). Bridging the intention - behaviour gap: Planning selfefficacy, and action control in the adoption and maintenance of physical exercise. Psychology \& Health, 20(2), 143-160. https://doi.org/10.1080/08870440512331317670

The British Psychological Society. (2019). Psychological perspectives on obesity: addressing policy, practice and research priorities. https://www.bps.org.uk/sites/bps.org.uk/files/Policy/Policy Files/Psychological Perspectives on Obesity - Addressing Policy\%2C Practice\%2C and Research Priorities.pdf

Thornicroft, G. (2006). Shunned: Discrimination against People with Mental IIIness. Oxford University Press.

Webb, J., Hall, J., Hall, K., \& Fabunmi-Alade, R. (2016). Increasing the frequency of physical activity very brief advice by nurses to cancer patients. A mixed methods feasibility study of a training intervention. Public Health, 139, 121-133. https://doi.org/10.1016/j.puhe.2016.05.015

West, R., \& Michie, S. (2020). Routes of transmission of SARS-CoV-2 and behaviours to block it: a summary. Qeios, 5-8. https://doi.org/10.32388/f6m5cb.2

World Health Organization. (2020a). Coronavirus disease 2019 ( COVID-19) Situation report 51.

World Health Organization. (2020b). Statement - Behavioural insights are valuable to inform the planning of appropriate pandemic response measures. https://www.euro.who.int/en/mediacentre/sections/statements/2020/statement-behavioural-insights-are-valuable-to-inform-the-planning-ofappropriate-pandemic-response-measures

YouGov. (2020). Personal measures taken to avoid COVID-19. https://yougov.co.uk/topics/international/articles-reports/2020/03/17/personal-measures-taken-avoid-covid19 
